\title{
Reasons for failure of antihypertensive treatment
}

\author{
O OGUNYEMI
}

\begin{abstract}
One hundred patients whose hypertension was originally well controlled were carefully screened when a routine clinic visit showed that their blood pressure was above $170 / 100 \mathrm{~mm} \mathrm{Hg}$. Simple misconceptions accounted for 75 failures: 38 did not know they had to continue their drugs, 14 thought they should not take antihypertensive drugs if they had not had a meal, 13 did not know which drugs controlled their blood pressure, and 10 believed it was better not to take their drugs on clinic days.

Eleven patients were using racemic alpha-methyldopa, which was ineffective; 11 others said they could not afford the drugs; only three intentionally stopped their drugs because of unpleasant side effects.

Patients need to be thoroughly informed about their treatment and the number of drugs kept to a minimum.
\end{abstract}

\section{Introduction}

Hypertension may fail to respond to treatment for many reasons. The patient may be unreliable in taking drugs either regularly or as prescribed. He may be too poor to be able to afford them. He is often ignorant of the fact that treatment is life long and may stop it when symptoms are relieved. Injudicious advice is sometimes given by a doctor to stop treatment on the basis of a single normal blood pressure measurement. The patient himself may not know what is important among several prescribed drugs. If they have been given for short periods in hospital he may erroneously assume that treatment was only for the period of time they lasted.

There are errors of commission too. The drug may not be prescribed in adequate dose or frequency; the hypertension may not be responsive to a particular drug or combination of drugs. Occasionally one proprietary preparation is effective while another, with the same generic formulation, is not. This last observation led me to study the causes of treatment failure in patients with hypertension seen at the General Hospital, Lagos.

\section{Patients and methods}

One hundred consecutive outpatients who were already being treated for hypertension were screened when their blood pressure on the day of attendance was not within acceptable controlled values. They had all been previously well controlled for at least six months or on six different occasions. Those who had never been properly controlled were excluded. Blood pressure was measured with the

Lagos General Hospital, Nigeria, West Africa

O OGUNYEMI, MB, BS, MRCP, chief consultant physician subject sitting and standing, and if there was any major difference between the two readings, the standing pressure was used. Diastolic pressure was recorded as the point of sound disappearance (Korotkoff phase V) or sound muffling (Korotkoff phase IV) ${ }^{1}$ when the former appeared inconsistent or difficult to identify.

If blood pressure on the day of attendance was $170 / 100 \mathrm{~mm} \mathrm{Hg}$ or more the patient was asked: Are you taking your drugs? If the answer was yes the following questions were asked in sequence: (a) Can you name your drugs ? (b) Can you describe them ? (c) Do you know their function? (d) When and how often do you take them? (e) How do you ensure a continuous supply ? $(f)$ Do you know how long you have to take them for? and $(g)$ Are you taking drugs not prescribed by the clinic?

If the answer was no the questions were: $(a)$ When did you last take any drugs? (b) Why have you not been taking them? (c) If you have finished them why did you not get some more? (d) Do you know why you have to take them ? $(e)$ Do you know how long you have to take them for ? and $(f)$ Are you taking drugs not prescribed from the clinic?

\section{Results}

The 100 patients were aged between 30 and 90 years (mean 53 years). The mean age of the 67 women was slightly lower (46 years) than that of the 33 men $(62.2$ years). Duration of treatment before failure ranged from two months to nine years, with an average of nine months, but more than $80 \%$ of all failures occurred within the first year of clinic attendance.

About $60 \%$ of the women, although married, had an independent but largely inadequate means of livelihood, mostly by petty trading or working as cleaners in offices. Two thirds earned less than $£ 840$ a year. The remainder were dependent on husbands or relations, most of whom earned between $£ 1680$ and $£ 2500$ a year. Nearly $90 \%$ of all women and over $80 \%$ of men were in the lower socioeconomic groups.

\section{CAUSES OF FAILURE}

Fifty one patients admitted having some drugs: 25 had the full complement of prescribed antihypertensive drugs, 15 had some of them, and 11 had drugs which were irrelevant to treatment of hypertension, such as antidiabetic tablets, analgesics, vitamins, and haematinics.

Twenty four patients had taken drugs that morning, including 11 who took all the prescribed antihypertensive agents and seven who took only part of their treatment because some drugs were finished; the remaining six patients took various drugs with no antihypertensive effect.

Twenty seven patients did not take any drug that morning, although they had some, (a) because they had not had a meal, having left home early to attend the clinic (14), (b) because they thought they should not take drugs when coming to the hospital (11), or (c) because of intolerable side effects (3).

The 11 patients who took all their antihypertensive drugs and yet did not achieve adequate control had prescriptions for Aldomet (methyldopa) $250-500 \mathrm{mg}$ three or four times daily, Moduretic (hydrochlorothiazide $50 \mathrm{mg}$ and amiloride hydrochloride $5 \mathrm{mg}$ combined) 1-2 tablets in the morning, and Valium (diazepam) 
$10 \mathrm{mg}$ at night. The dose of methyldopa in the first four patients had already been increased several times, and a change to another drug was being contemplated. At this stage it was noticed that a generic methyldopa with a dusky yellow colour was being used and not Aldomet. When the bright yellow Aldomet tablet inscribed MSD was substituted, adequate control of blood pressure was achieved within two weeks.

Of the 49 patients who had no drugs, 11 said drugs were not supplied and they could not afford to buy them, and 38 did not know they had to use more drugs once their supply was finished.

\section{Discussion}

Little is known about why Nigerians whose hypertension was once controlled later lose control. The impression gained from most authors is that economic factors must be dominant, ${ }^{2-4}$ whereas those from the West make a case for non-compliance, due to such factors as frequency of administration, intolerable side effects, and drug resistance. ${ }^{5-10}$

Only three of our patients in this study stopped their drugs because of side effects: extreme weakness in one woman and impotence in two men. In $75 \%$ failure of compliance was due to simple misconceptions which could easily have been avoided by proper information. Thirty eight patients did not know they had to continue their drugs; 14 thought they should take the drugs only if they had had a meal; and 13 were unaware which drugs were necessary to control blood pressure.

Simple information together with an explanation of the dangers of untreated hypertension were enough to persuade both the patients with side effects and those who claimed they could not afford drugs to get their drugs regularly and to take them.

Polypharmacy, often with unnecessary drugs, accounted for non-compliance in a few patients, and this is particularly undesirable for patients who have to take drugs for life. Pre- scriptions should be kept to a minimum. The dusky yellow methyldopa tablet turned out to be racemic alpha-methyldopa, with no indication of the ratio of laevo to dextrorotatory isomers, of which only the former is antihypertensive. ${ }^{11}$

It is of great concern that 20 years after Aldomet became available unreliable racemic alpha-methyldopa should still be allowed to be dispensed. This hospital pharmacy has since been instructed to stock only Aldomet (MSD), and the two types of methyldopa are shown to each patient and the difference explained.

\section{References}

${ }^{1}$ Korotkoff NS. On methods of studying blood pressure. Izv Voenno-med Akad 1905;11:365-7.

${ }^{2}$ Ribeiro MB. Hypertension and economic activities in Sao Paulo, Brazil. Hypertension 1981;11:233-7.

${ }^{3}$ De Aquiar JH. Treatment of hypertension in low income population. Arq Bras Cardiol 1981;36:301-3.

${ }^{4}$ Crutchfield SE. Hypertension costs: an insurance perspective. Natl Med Assoc 1980;72:523,526.

${ }^{5}$ Gillum RF. Diagnosis and management of patient noncompliance. fAMA 1974;228:1563.

${ }^{6}$ Hollifield JW, Stanton PE Jr. Effectiveness of twice daily methyldopa in essential hypertension. $\mathcal{F}$ Tenn Med Assoc 1978;71:337.

${ }^{7}$ Duke M. Thiazide induced hypokalemia associated with myocardial infarction and ventricular fibrillation. $\mathcal{F} A M A$ 1978;239:43-5.

${ }^{8}$ Felts JH, Charles J. Minoxidil in refractory hypertension. $\mathcal{F}$ Cardiovasc Pharmacol 1980;2:52.

9 Wells JO. Unusual cases of resistance to minoxidil therapy. $\mathcal{f}$ Cardiovasc Pharmacol 1980;2:52.

${ }^{10}$ Atkinson AB, Brown JJ, Lever AF, Robertson JIS. Combined treatment of severe intractable hypertension with captopril and diuretic. Lancet 1980 ;ii : $105-8$.

11 Oates JA. The discovery of methyldopa's antihypertensive effect and initial pharmacologic investigations in man. In: Zanchetti A, ed. Methyldopa in hypertension. New Jersey: Merck \& Co, 1978.

(Accepted 10 March 1983)
Harrison's Principles of Internal Medicine states: "The platelets of migraine patients are deficient in monoamine oxidase responsible for degrading phenylethylamine." What laboratory test is necessary to discover if platelets are indeed deficient in monoamine oxidase?

In 1974 Sandler $e t$ al reported on the results of their investigation of 28 migrainous subjects. ${ }^{1}$ The unselected group comprised nine male and 19 female subjects and during a headache free period, venous blood samples were collected into plastic tubes containing lithium heparin. Platelets were separated and stored deep frozen before assay. Non-migrainous volunteers of a similar age range provided control samples. Monoamine oxydase activity was measured radiometrically using $\mathrm{C}$ labelled phenylethylamine, tyramine, dopamine, and 5hydroxytryptamine as substrates. A highly significant decrease in phenylethylamine oxidising ability was observed in migrainous compared with control subjects. In addition, they reported a difference in tyramine oxidation of a similar order and also a smaller decrease in dopamine oxidation.-KEVIN ZILKHA, consultant neurologist, London.

' Sandler M, Youdim MBH, Hanington E. A phenylethylamine oxidising defect in migraine. Nature 1974;250:335-7.

\section{Is there any link between bacteriuria in pregnancy and the development} of oedema?

Both conditions are common. Asymptomatic bacteriuria occurs in $5-7 \%$ of pregnant women, ${ }^{12}$ and about $80 \%$ of healthy pregnant women have clinically detectable oedema. ${ }^{3}$ Women with bacteriuria have an increased risk of developing acute pyelonephritis and are said to have increased risks of impaired renal function, miscarriage, hypochromic anaemia, hypertension, low birth weight, and fetal mortality. ${ }^{24}$ There is, however, controversy about some of these associations-for example, some studies ${ }^{1}$ have failed to show a link with pre-eclampsia. Although mild oedema is now regarded as a good prognostic sign in pregnancy, ${ }^{3}$ severe generalised oedema may be associated with fulminating pre-eclampsia or (rarely) renal disease, and these two conditions form the only links, so far as I know, between bacteriuria and oedema. A link of a different type is that both bacteriuria and pre-eclampsia may cause proteinuria, and when proteinuria is detected the woman must have her blood pressure checked and must have a bacteriological check on her urine.-JAMES OWEN DRIFE, senior lecturer in obstetrics and gynaecology, Leicester.

1 Bailey RR. Bacteriuria of pregnancy. NZ Med f 1982;95:56

McFadyen IR. Pregnancy bacteriuria and Escherichia coli. $\boldsymbol{f} R$ Soc Med 1980;73: 227-9.

Robertson EG. The natural history of oedema during pregnancy. Fournal of Obstetrics and Gynaecology of the British Commonevealth 1971;78:520-9.
Gilstrap LC, Leveno KJ, Cunningham FG, Whalley PJ, Roark ML. Renal infection and pregnancy outcome. Am f Obstet Gynecol 1981;141:709-16.
int

Babies and young children often develop a rash during treatment with penicillin $V$ or amoxycillin. It is sometimes difficult to decide whether this is a viral rash or due to the treatment. Should poliomyelitis vaccine then be withheld from these children or, if given inadvertently, would the result be the recurrence of the rash, or could a more serious reaction occur?

In these circumstances, the benefits of oral polio vaccination would seem to outweigh greatly the risk of allergic reactions to penicillin that has remained in the vaccine from the original seed culture. The maximum amount of penicillin present in the vaccines marketed in Britain is 0.04 units per dose and the chance of an untoward reaction to such a small dose would seem to be remote, particularly when this dose is compared with a starting dose more than 10 times greater than is used in a relatively conservative penicillin desensitisation regimen, although antihistamine cover would be given in the latter case. To my knowledge, the only well documented report of an allergic reaction to penicillin after polio vaccine given to a 43 year old woman resulted in a relatively mild reaction on each of two occasions when the vaccine was given after earlier penicillin exposure. ${ }^{2}$ Polio vaccine should be given in the circumstances described and in the rare event of reaction a mild rash with urticaria is the most probable clinical finding. - G RYLANCE, consultant in paediatric clinical pharmacology, Birmingham.

Fellner MJ, Van Hecke E, Rozan M, Baer RL. Mechanisms of clinical desensitisation in urticarial hypersensitivity to penicillin. $\mathcal{F}$ Allergy 1970;45:55-61. Ducos J, Colombies P, Ohayon E, Toulouse F. Administration orale de vaccin antipoliomyélitique. Réaction d'hypersensibilite conséquente. Intérêt du tes de transformation blastique pour le diagnostic étiologique. Presse Med 1968;76:
2347-8. 